Review

\title{
Lactate as a Regulator of Cancer Inflammation and Immunity
}

\author{
Nuno Santos ${ }^{1,2}$, Andreia Pereira-Nunes ${ }^{1,2}$, Fátima Baltazar ${ }^{1,2}$, \\ Sara Granja ${ }^{1,2, *}$ \\ 1 Life and Health Sciences Research Institute (ICVS), School of Medicine, \\ University of Minho, Braga 4710-057 Portugal \\ 2 ICVS/3B’s-PT Government Associate Laboratory, Braga/Guimarães, 4710-057 \\ Portugal \\ * Correspondence: Sara Granja, Email: saragranja@med.uminho.pt; \\ Tel.: +351-253604868.
}

\begin{abstract}
Resistance to anti-cancer therapies is a consequence of adaptation of cancer cells but also of maladaptation of tumor-infiltrating immune cells. The opposing roles acquired by the immune system have to be faced in order to fight tumor growth and therapy resistance. Effector immune cells are recruited and activated but they are blocked by the strong immunosuppressive nature of the tumor microenvironment (TME). Immune evasion and deregulation of energy metabolism are two hallmarks of cancer that may be functionally linked. Malignant cells which present a high glycolytic phenotype, besides creating metabolic demanding environments that encroach on the function of tumorinfiltrating immune cells, also release immunosuppressive metabolites and by-products, such as lactate, forming a metabolic symbiosis with immune cells. This acidic TME has a strong impact in the profile of tumorinfiltrating immune cells, being instrumental for immunosuppression. Therefore, in this review, we focus on key molecular mechanisms by which lactate metabolically modulates immune cell response during tumor development and progression.
\end{abstract}

KEYWORDS: lactate; immune evasion; tumor microenvironment; metabolic crosstalk

Received: 20 August 2019

Accepted: 27 September 2019

Published: 16 October 2019

Copyright (C) 2019 by the author(s). Licensee Hapres, London, United Kingdom. This is an open access article distributed under the terms and conditions of Creative Commons Attribution 4.0 International License.

\section{INTRODUCTION}

\section{Crosstalk between Cancer Inflammation and Metabolism}

The first association between inflammation and cancer was documented in 1863 by Rudolf Virchow when he detected leukocytes in tumor tissues and made the connection between cancer and inflammation [1]. This hypothesis gained significant attention in the last few years leading to the recognition of the tumor-associated inflammation as a key hallmark of cancer [2,3]. Inflammation is characterized by a complex biological response to cellular damage, where the immune 
system attempts to eliminate and neutralize the injury and initiates the regenerative and healing processes [4,5]. Commonly, inflammation is classically viewed as a feature of the innate immune system that could be recruited during tumor initiation or development and, according to numerous reports, elevated inflammatory mediators are associated to poor prognosis in cancer patients [5,6]. Tumor cells produce various cytokines and chemokines that attract leukocytes into the tumor niche, facilitating inflammation-mediated tumorigenesis. Likewise, prolonged inflammation triggers altered expression of oncogenes and tumor suppressor genes that will promote tumor aggressiveness [7]. Immune cell activation leads to important alterations in several signaling pathways in order to exert their effector function. This process is metabolically challenging as widely described, and therefore, immune cells reprogram their metabolism to sustain their energetic and metabolic needs. In the last years, immunometabolism has become one of the most exciting areas of translational research. Indeed, understanding how such metabolic pathways determine specific immune cell fate and the functional responses is crucial to understand and target cancer immune evasion [8].

Another characteristic feature of tumor cells is deregulated metabolism. Indeed, cancer metabolism has emerged as an area of research in cancer biology that has increasingly gained attention and was also recognized as a hallmark of cancer [2,3]. This field allowed to understand the processes involved in malignant transformation and also identified suitable therapeutic targets that are altered in cancer cells [9]. In fact, in the 1920s Otto Warburg described that cancer cells display a glycolytic phenotype, with increase rates of glucose consumption, and consequently increase in lactate production regardless of oxygen availability [10,11]. In order to maintain $\mathrm{pH}$ homeostasis and avoid glycolysis inhibition due to a negative feedback mechanism, cells export lactate and protons into the extracellular milieu via Monocarboxylate Transporters (MCTs) [12]. The accumulation of lactate and protons into the extracellular space induces a drop in the extracellular $\mathrm{pH}$, acidifying the surrounding environment [13-15]. As for cancer cells, immune cells also adapt their metabolic status as a consequence of changes in the surrounding microenvironment [16]. Effective immune cells have high metabolic demands for their activation and differentiation, while resting immune cells generate most of their energy from fatty acid oxidation (FAO) or from tricarboxylic acid (TCA) cycle, which is linked to the generation of ATP via oxidative phosphorylation (OXPHOS) [17,18], to maintain their housekeeping functions.

Previously considered as a waste product of anaerobic metabolism in cancer, lactate represents an important signaling molecule involved in sophisticated mechanisms that shutdown anti-tumor immune responses and activate potent negative regulators of adaptive and innate immunity [19]. This review summarizes the key molecular mechanisms by which 
lactate metabolically modulates the immune cell response during tumor development and progression.

\section{LACTATE IN THE TUMOR MICROENVIRONMENT}

Several evidence have shown that intermediate or end products of metabolic pathways not only function as feedback regulators and provide substrates to be used by other pathways, but also bind to cognate receptors to initiate de novo signaling cascades. The glycolysis end-product lactate, a 3-carbon hydroxycarboxylic acid, is an essential metabolite that circulates at levels of 1-2 mM and acts as an inter-organ carbon shuttle in mammals $[20,21]$ rising to $10 \mathrm{mM}$ and even to $30-40 \mathrm{mM}$ in the tumor microenvironment (TME) [22-24] and has been greatly associated with cancer aggressiveness (reviewed in [25]). Proton-coupled lactate efflux from cancer cells is an important player in the maintenance of the cancer acidic phenotype and contributes to several features of tumor progression by modulating the TME [2], including cell migration and invasion, angiogenesis, and escape to immune surveillance. As a consequence of high glycolytic flux or hypoxia, lactate is produced in the cytosol via the enzymatic activity of lactate dehydrogenase A (LDHA) [26]. LDHA is responsible for the rapid conversion of pyruvate into lactate with regeneration of $\mathrm{NAD}^{+}$, supporting the high glycolytic rates of cancer cells in which lactate is transported across the plasma membrane through MCTs and accumulated in the extracellular space. The first four isoforms (slc16a1/MCT1, slc16a7/MCT2, slc16a8/MCT3 and slc16a3/MCT4) were functionally validated to mediate the proton-linked plasma membrane transport of monocarboxylates such as lactate, pyruvate and ketone bodies [12]. MCT1-4 display distinct affinities for monocarboxylic acids which are associated with the expression patterns of these transporters within tissues (reviewed in [27]). However, given their physiological expression, МСT2 exhibits the highest affinity for monocarboxylates, followed by MCT1 and then MCT4. Extracellular lactate levels can be sensed by several cell types via lactate receptors such as $G_{1}$-proteincoupled receptors 81 and 132 (GPR81 and GPR132), hence modulating their function and metabolism [28,29]. Targeting lactate production, via LDHA, or transport, via MCTs has become a promising therapeutic opportunity in oncology. The discovery of an acidic $\mathrm{pH}$ in inflammatory and tumor sites aroused the interest of potential effects of lactate on cellular metabolism that might contribute to the modulation of immune cell function during inflammation in the TME. Several studies have highlighted the molecular mechanisms that govern the crosstalk between lactate metabolism and the immune system in tumors, and the impact of lactate in immunosuppression has been widely explored [22,30-34]. Although the knowledge on the molecular pathways underlying lactate-related immunomodulation in autoimmunity is just emerging, preliminary evidence indicates that extracellular lactate levels directly influence immune cell metabolism and cytokine production and may serve as a 
negative feedback signal limiting inflammation [16]. Moreover, TME is highly heterogeneous, with both oxygenated and hypoxic areas, forcing tumor and stromal cells to adapt to the environment in order to survive [35]. Thus, highly glycolytic malignant cells, besides creating metabolic demanding environments (low glucose) that encroach on the metabolism and function of tumor-infiltrating immune cells, also release immunosuppressive metabolites and by-products (lactate) forming a metabolic symbiosis/parasitism with immune cells [36,37]. Surprisingly, shuttling of metabolites has been described as a new route that cancer cells use to evade the immune system [38]. The mechanism by which lactic acid influences immunosuppression is not fully understood however, it is thought that, on one hand, high concentrations of lactic acid exported by cancer cells block the export of lactic acid by glycolytic immune cells and, therefore, disturb their metabolism and function [39]; on the other hand, immune cells consume lactate as an energy source, which will impair the glycolytic flux necessary for the activated phenotype [40] and acts as signaling molecule [39]. In this context, lactate fuels oxidative cells in the TME [41], being this symbiotic association mediated by MCT4 (preferentially mediates lactate efflux-present in highly glycolytic cancer cells) and MCT1 (preferentially mediates lactate uptake-present in stromal oxidative cells) [42]. The effect of lactate on inflammation was reported in acute pancreatitis and hepatitis models. The interaction between lactate and GPR81 in monocytes and macrophages, suppressed TLR4 and TLR9 mediated pro-inflammatory cytokine IL-1 $\beta$ and inflammasome components Nlrp3 and Casp1, via downregulation of NF$\kappa B$ [43]. Similarly, in a murine induced colitis model, lactate treatment prevented the rise of IL-6 serum levels, microbial translocation from the gut to liver and increased lactate concentration in the colon alleviates colitis [44]. The same group also demonstrated that lactate does not only downregulate TLR-mediated pro-inflammatory responses in macrophages and dendritic cells, but also in intestinal epithelial cells [45].

Interestingly, results from murine in vitro and in vivo models suggest that glucose deficiency and lactate accumulation in the TME promote hurtful effects on the immune cells that were poised to infiltrate and destroy tumors [46]. It has been reported that carcinomas overexpressing LDHA have poor lymphocyte response. Also, it was shown that LDHAmediated production of lactate by melanoma tumor cells and subsequent TME acidification inhibits tumor surveillance by $\mathrm{T}$ and NK cells resulting in diminished IFN- $\gamma$ production and tumor immune escape [47]. To date, several studies demonstrated strong effects of lactate and lactic acid on immune cell populations in vitro and in vivo as will be documented below.

\section{LACTATE IN INNATE IMMUNITY IN CANCER}

The interaction between innate and adaptive immune cells is crucial to regulate an effective immune response, and a wide variety of immune cells can be found in the TME. At the early beginning of inflammation, 
neutrophils are the first cells to infiltrate upon inflammatory mediators released at the site of inflammation [5]. The importance given to neutrophils in cancer has increased in the last years. Indeed these cells have been detected already in a wide variety of human cancer types, including lung, breast and gastric cancers, melanoma, and others [48-50]. However, the role of tumor-associated neutrophils (TANs) in cancer is still controverse. While there is some evidence that TANs release cytokines and chemokines able to eliminate and fight cancer cells, other studies showed that these cells also have a prominent role in cancer immune evasion [51]. However, information about how lactate from the TME modulates TANs function is still scarce. It is described that lactate interfers with bone marrow vascular permeability reducing neutrophil mobilization through a GPR81 signaling dependent way [52]. Moreover, since neutrophils are best known as glycolytic cells, one may assume that lactate will have an impact in the metabolic profile of these cells and this metabolic reprogramming could prevent neutrophil effector functions.

Among innate immune cells, myeloid cells, namely macrophages were the first cells to be described in human tumors [53] and can comprise up to half of the tumor-infiltrating immune cells [54], where they become tumor-associated macrophages (TAMs). Macrophages are known to present high functional plasticity, with the ability to express distinct functional programs in response to different stimuli [55,56]. The classically activated macrophages with a pro-inflammatory phenotype (M1-like) are responsible for the clearance of pathogens, with the production of inflammatory cytokines and high antigen presentation. However, they have a controversial role in tumor development. While some studies suggest that these M1-like macrophages display anti-tumor characteristics, others showed their contribution in tumor initiation by secretion of chemokines and cytokines such as IL-6, TNF, IL-1 and iNOS [57]. On the other hand, alternatively activated macrophages, with an anti-inflammatory phenotype (M2-like) participate in angiogenesis and tissue remodeling and repair, displaying pro-tumor characteristics [58]. Emerging evidence reveals that M1-like and M2-like macrophages engage distinct metabolic demands. For instance, M1-like macrophages enhance their anabolic metabolism, including anaerobic glycolysis, pentose phosphate pathway activation and fatty acid synthesis while, M2-like macrophages rely on OXPHOS to support their metabolic demands. These metabolic alterations offer checkpoints to fine-tune macrophage behavior and strongly influence their functions in the TME [46].

Monocytes are leukocytes generated primarily in the bone marrow but can also be found in the blood and spleen. Monocytes circulate in the bloodstream and differentiate into macrophages or dendritic cells in response to chemotactic signals from different tissues. They are responsible for phagocytosis, antigen presentation and production of cytokines [59]. To date, several studies show the effect of lactate on monocytes and macrophages in vitro. High levels of lactate $(20 \mathrm{mM})$ at a 
$\mathrm{pH}$ of 7.4 inhibited monocyte migration in the Boyden chamber system [60]. Moreover, when cultured in the presence of lactate, monocytes and macrophages produce less pro-inflammatory cytokines and chemokines and decrease their glycolytic rates [33,60,61]. For instance, TNF secretion was suppressed by lactic acid in a co-culture model of human monocytes with multicellular tumor spheroids [61]. Also, lactic acid secreted by tumor cells activate the IL-23/IL-17 [62,63] proinflammatory pathway but not the Th1 pathway [63]. After activation, both monocytes and macrophages rely on glycolysis to support their function [33,61]. In order to sustain glycolysis, lactate should be exported, however, extracellular acidification reduces the export of lactate by monocytes and macrophages, impairing glycolysis and consequently the expression of pro-inflammatory mediators [61,64]. Lactate can also have a signaling role in driving cancer immune evasion, as it was shown to induce M2-like macrophage polarization by activating the ERK/STAT3 signaling pathway [65], although others have proposed that high levels of tumor-derived lactate drives M2-like macrophage polarization through stabilization of HIF-1a, increasing the levels of Arginase 1 (ARG1) and vascular endothelial growth factor (VEGF) [66]. Ohashi and co-workers showed that dichloroacetate (DCA), an inhibitor of pyruvate dehydrogenase kinase, suppresses the activation of the IL-23/IL-17 pathway and the expression of ARG1 in TAMs, induced by lactic acid. Importantly, tumor-bearing mouse spleen treated with DCA decreased ARG1 expression in tumor-infiltrating immune cells and increased the number of IFN- $\gamma$-producing $\mathrm{CD}^{+} \mathrm{T}$ cells and NK cells [67]. GPR132 ( $\mathrm{G}$ protein coupled-receptor 132), a lactate receptor/sensor highly expressed in macrophages, promotes the M2-like phenotype, facilitating breast cancer metastasis [29]. Lactate can also inhibit pro-inflammatory responses in macrophages in a GPR81 (G protein coupled-receptor 81)independent manner [33]. A recent study explored the relationship between lactic acid concentration and M2-like macrophage polarization in biopsies from patients with head and neck squamous cell carcinoma (HNSCC), by measuring the expression of M2 macrophage markers, CSF1R and CD163. Tumors with high lactic acid concentration showed higher levels of CSF1R and CD163 expression, suggesting that tumor-derived lactic acid promotes M2-like macrophage polarization in human HNSCC [68]. Furthermore, lactate derived from a pancreatic tumour cell line induced polarization of THP1 (human monocytic cell line) into an M2-like phenotype [69]. In a microfluidic device, lactate exported by bladder cancer cell lines reprogrammed TAMs to an M2-like phenotype, while blockage of MCTs interrupted the lactate shuttle, and consequently inhibited the M2-like phenotype [70]. In summary, these data show that tumor-derived lactate skews macrophages towards a pro-tumoral phenotype.

Dendritic cells (DCs) are professional antigen presenting cells (APCs), capable of capturing, processing and presenting antigens to $\mathrm{T}$ cells, 
initiating primary T-cell responses. Besides linking innate and adaptive immunity, DCs promote tolerance to self-antigens, minimizing autoimmune reactions [71]. Upon resting state, DCs exhibit an immature phenotype, but upon antigen encounter, undergo a process of "maturation", where they become activated and enhance the expression of costimulatory molecules (CD80/CD86), major histocompatibility complex II (MHC-II), production of cytokines such as IL-12 and increase glycolytic rates. Once activated, DCs migrate to lymph nodes, presenting antigens to antigen-specific adaptive immune cells ( $T$ and $B$ cells) [72]. Infiltration of DCs in the tumor microenvironment, where they become tumor associated dendritic cells (TADCs), positively correlates with patient survival and improved prognosis. However, their role in cancer progression is still debatable, since studies demonstrate that TADCs express low levels of costimulatory molecules, low production of IL-12 and limited antigen-presenting capacity [73]. High levels of lactate cause acidification of the tumor microenvironment, and there is opposing data regarding the effect of lactate itself or low extracellular $\mathrm{pH}$ on the functionality of DCs. In vitro studies show that acidification of the culture medium by addition of $\mathrm{HCl}$ enhanced endocytosis and increased the expression of MHC-II, CD11C and costimulatory molecules CD80 and CD86 by DCs [74], while addition of lactate to culture medium reduced the expression of CD1a, CD83 and HLA-DR. Besides, DCs cultured with lactate decreased the production of IL-12, increased production of IL-10 and displayed reduced migratory capacity. In a murine glioma model the treatment with diclofenac, a LDHA inhibitor, reduced intratumoral lactate levels that resulting in reactivation of DCs toward Toll-like receptor (TLR) stimulation, which inhibited accumulation and activation of Tregs [75]. Furthermore, blockade of lactic acid production in melanoma and prostate multicellular tumor spheroids co-cultures reverted the TADC phenotype to normal [76,77]. This raises the possibility that low extracellular $\mathrm{pH}$ facilitates the impact of lactate on DC activity. As it is well described, MCTs transport lactate coupled with protons following a concentration gradient [15]. In this sense, the high concentration of lactate in the tumor microenvironment, produced by hyperglycolytic cancer cells, blocks the export of lactic acid from DCs necessary to sustain the high glycolytic rates of activated DCs and thus hinder their metabolism and function.

Natural killer (NK) cells are cytotoxic lymphocytes of the innate immune system, which display activity against cells under stress such as tumor cells. NK cells present both activating and inhibitory receptors. Healthy cells express major histocompatibility complex I (MHC-I) on their surface, which is an inhibitory signal for NK cells. However, tumor cells lose MHC-I expression, therefore the signal from inhibitory receptors is diminished, activating NK cells to eliminate tumor cells directly through exocytosis of granules containing proteases (known as granzymes), or indirectly through secretion of cytokines such as IFN- $\gamma$. NK cells also 
interact with other immune cells to regulate their activity [78]. Similar to other immune cells, NK cells are also affected by lactate. Lactate inhibits NK cell activity, by downregulation of the activating receptor NKp46 and inhibiting the secretion of granzymes. Furthermore, this immunosuppressive effect of lactate was enhanced in a low extracellular $\mathrm{pH}$ environment [79]. Another study demonstrates that tumor derived lactate inhibits NK cell activity, in which tumors with reduced lactate production showed increased infiltration of NK cells. Exposure to lactate caused NK cell apoptosis and interfered with the regulation of nuclear factor of activated T cells (NFAT), reducing production of IFN- $\gamma$ [47].

Myeloid-derived suppressor cells (MDSCs) are a heterogeneous population of immature immune cells from the monocytic and granulocytic pathway, that expand in pathological conditions such as chronic inflammation and cancer. MDSC generation and accumulation is caused by cancer cells overexpressing colony-stimulating factors, leading to deregulated hematopoiesis. MDSCs promote tumor growth and mediate immune tolerance, as MDSC activity was originally associated with T cell immunosuppression, however, recent studies demonstrate that it also interacts with macrophages, DCs and NK cells [80,81]. LDHA knockdown in pancreatic cancer cells decreases the number of MDSCs in the tumor niche [79]. Tumor-derived lactate promotes MDSC proliferation and survival, which inhibits NK cell activity [47]. This evidence suggests that lactate can promote an immunosuppressive environment indirectly through other immune cells.

\section{LACTATE IN ADAPTIVE IMMUNITY IN CANCER}

Adaptive or acquired immunity is the second line of defense of the immune system against non-self pathogens, also referred as specific immunity. It is mediated by $\mathrm{T}$ and $\mathrm{B}$ lymphocytes and its role is to recognize and eliminate non-self antigens during the process of antigen presentation, and develop immunological memory [82]. T cells are grouped in two major subsets, $\mathrm{CD} 4^{+} \mathrm{T}$ Helper cells (Th) and $\mathrm{CD} 8^{+}$Cytotoxic $\mathrm{T}$ cells (CTL). Th cells mediate the acquired immune response, by the release of a plethora of cytokines that influence the activity of other immune cells. Th cells can have 4 distinct fates, determined by different signals upon interaction with antigens. These are Th1, Th2, Th17 and Treg cells. Th1 cells initiate Th1 immune response, which is more effective against intracellular bacteria, is characterized by the release of cytokines such as IFN- $\gamma$ and IL-2 and being the effector cells macrophages and CD ${ }^{+}$ $\mathrm{T}$ cells, while Th2 cells initiate a Th2 immune response, more effective against extracellular parasites, characterized by the production of IL-4 and IL-10 by the effector cells eosinophils, basophils and B cells [83]. Th17 cells produce IL-17 and mediate immune responses against extracellular bacteria [84,85]. Treg cells participate in the suppression of the immune response, by the production of IL-10 and TGF- $\beta$ [83]. CTLs are T lymphocytes that kill cancer cells that are either damaged or infected. 
CTLs recognize specific antigens bound to MHC-I molecules in antigen presenting cells through their TCRs (T-cell receptors). Once activated, CTLS undergo clonal expansion, proliferate rapidly and travel throughout the body searching for cells that carry that specific antigen, to eliminate them [82]. B lymphocytes circulate in blood plasma and lymph and are responsible for the production of antibodies. Once B cells recognize an antigen through their BCRs (B-cell receptors), and receive additional signals from Th cells, they differentiate into plasma cells, capable of secreting antibodies against that unique antigen [86].

Upon antigen presentation, activated $\mathrm{T}$ cells undergo metabolic reprogramming and increase their glycolytic flux, which is essential for their proliferation and effector functions. Effector T cells and Th17 cells rely on aerobic glycolysis, while Treg cells rely on oxidative phosphorylation to produce ATP [87]. Acidity of the extracellular environment has been reported to have an impact on $\mathrm{T}$ cell activity. Lactate levels are increased in inflammatory pathologies [22,88]. Lactate accumulated in the synovial joints of rheumatoid arthritis (RA) patients, as LDHA and MCT4 activity was increased in RA synovial tissue [16]. Sodium lactate interacts with the transporter SLC5A12 on the surface of $\mathrm{CD}^{+} \mathrm{T}$ cells, leading to impaired migration, downregulation of glycolytic enzymes and production of IL-17. Lactic acid interacts with the transporter MCT1 on the surface of $\mathrm{CD}^{+} \mathrm{T}$ cells, impairing migration and cytoxicity. Neither the presence of sodium lactate or acidification of the culture medium with $\mathrm{HCl}$ alone affected $\mathrm{CD} 8^{+} \mathrm{T}$ cell migration, suggesting that the presence of both lactate and $\mathrm{H}^{+}$is necessary to regulate $\mathrm{CD} 8^{+} \mathrm{T}$ cell motility. Decreased $\mathrm{T}$ cell motility, resulting in their retention in the synovial joint, coupled with increased production of IL-17 drives chronic inflammation in RA [22]. The first studies showing the effect of low $\mathrm{pH}, \mathrm{T}$ were cells cultured in medium containing $\mathrm{HCl}$ and they described the impairement of proliferation, cytotoxicity and cytokine production [89,90]. High lactate concentrations in the extracellular medium block the export of lactate by activated $\mathrm{T}$ cells, disturbing their metabolism and function [91]. Lactate suppresses proliferation and cytokine production of CTLs [47,91], by interfering with the TCR-triggered phosphorylation and activation of JNK/c-Jun and p38 pathways, blocking IFN- $\gamma$ production [92]. Moreover, Lactic acid suppressed the proliferation and cytokine production of human cytotoxic T lymphocytes (CTLs) up to 95\% and led to a 50\% decrease in cytotoxic activity. Importantly, blockade of MCT1 resulted in impaired CTL function. Since export of lactate is essential for proper clonal expansion of activated T cells [93], one can conclude that high lactic acid concentrations in the tumor environment block lactic acid export in T cells, thereby disturbing their metabolism and function [91].

However, neutralizing the extracellular $\mathrm{pH}$ abrogated the effect of lactate [89]. This demonstrates that acidification of the medium synergizes with lactate to promote an immunosuppressive environment. In addition, tumor-derived lactate downregulates the expression of FAK family- 
interacting protein FIP200, resulting in naive T cell apoptosis, autophagy impairment and consequently, poor antitumor immunity [94]. The acidic tumor microenvironment is permissive for the accumulation of Treg cells, as their frequency in tumor sites often correlates with poor prognosis in several cancers. Inhibition of glycolysis, as a consequence of high levels of extracellular lactate, increases the expression of transcription factor FoxP3, an important transcription factor for Treg cell function [92]. FoxP3 stimulates the oxidation of extracellular lactate to fuel mitochondrial activity, providing Treg cells with a metabolic advantage in high-lactate conditions [65]. This adaptation of Treg cells to acidic conditions potentiates their immune suppressive function. However, the effect of lactate in B cell function remains to be elucidated [47].

\section{CONCLUDING REMARKS}

Accumulating evidence support the idea that understanding how metabolism controls immune cell function could provide new therapeutic opportunities for the many diseases associated with immune system deregulation, including cancer. Definitely, both cancer and activated immune cells depend on aerobic glycolysis to fulfill their energy demands in order to proliferate and function. In a highly hostile TME, while on one hand cancer cells gobble glucose which will result in a competition for available nutrients affecting immune effector cell metabolism and consequently their function, proliferation, as well as differentiation, on the other hand, cancer cells will produce large amounts of lactate which will result in acidification of the TME. Indeed, lactate is reported to be one of the most important metabolite of the TME that modulates the metabolism of innate and adaptive immune system that either subverts the anti-tumorigenic functions toward pro-tumorigenic functions or enhances the immune suppressive functions thereby potentiating tumor progression [95] (Figure 1). Notably, since Tregs prefer oxidative metabolism to proliferate and survive, it is expectable that excess lactate will not negatively impact on Tregs metabolic profile and indeed can be used as energy source allowing their maintenance in the acidic TME [96].

Given the importance of lactate in the immunosuppressive phenotype of cancer cells, inhibition of lactate production could be one of the important strategies being considered for cancer therapy [97]. In line with this, Brand and co-workers showed that LDHA inhibition in cancer cells increased the infiltration of IFN- $\gamma$-producing $\mathrm{T}$ and $\mathrm{NK}$ cells and significantly decreased tumor growth [47]. Accordingly, blocking acidification prior to immunotherapy improved anti-tumor response [90]. Lactate transporters (MCTs) could be also potential targets to revert TME acidification and recover immune cell function. First, their inhibition in cancer cells will control the concentration of lactate in the TME and secondly, they are major players in the tumour metabolic symbiosis, namely lactate shuttling between cancer and stromal cells, which was already denoted by previous encouraging studies [98]. Blocking TME 
acidification could also be accomplished by inhibiting different $\mathrm{pH}$ regulators for e.g., V-ATPase and NHE1, or by the addition of bicarbonate into the TME [92]. Indeed, neutralizing tumor acidity with bicarbonate was associated with T-cell infiltration, with consequent decrease in tumor growth and, importantly, potentiated immunotherapies [99]. For instance, a promising class of ATPase inhibitors in cancer are the proton pump inhibitors (PPIs), that include omeprazole, esomeprazole among others. These compounds, already in clinical use for gastric acid control, have been successfully used to suppress tumor growth in vitro and in vivo [15]. Importantly, using esomeprazole to buffer TME improves $\mathrm{T}$ cell infiltration in the tumor mass and delayed cancer progression [90]. Interestingly, Cariporide, a specific and powerful NHE1 inhibitor, also promoted activation of pro-inflammatory TAMs and increased cytotoxic $\mathrm{T}$ cell infiltration into mouse glioma tumors [100]. The massive advances in stimulating anti-tumor immunity by checkpoint blockade raises significant questions about how tumors and the tumor microenvironment inhibit immune cell function and how this can be overcome. Therefore, development of therapies that block the acidification of TME aiming to recover effector and memory $\mathrm{T}$ cells and reduce suppressive functions of Tregs hold significant potential for cancer immunotherapy.

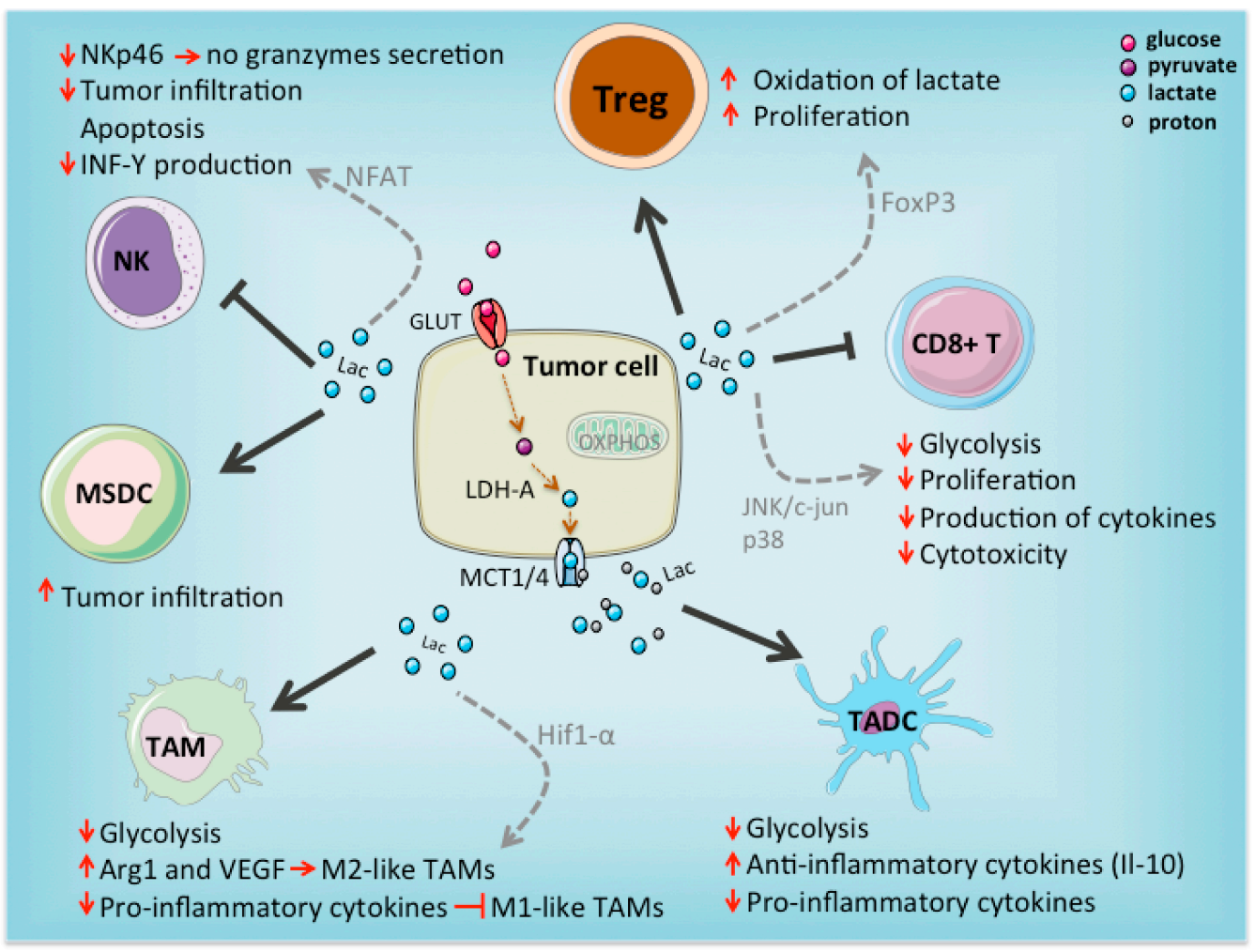

Figure 1. Impact of lactate in immunosuppression. Highly glycolytic cancer cells export lactate and induce TME acidification that strongly skewed immune response by altering tumor infiltrating immune cells. TAM: tumor associated macrophages; TADC: tumor associated dendritic cells; CD8 ${ }^{+} \mathrm{T}$ : cytotoxic T lymphocytes; NK: natural killer; Treg: T regulatory cells; MDSC: myeloid-derived suppressor cells; GLUT: glucose transporters; MCT1/4: monocarboxylate transporters 1/4; LDH-A: lactate dehydrogenase A; Lac: lactate; grey arrows: possible mechanism. 


\section{AUTHOR CONTRIBUTIONS}

All the authors jointly prepared the text, SG and FB developed the structure and arguments for the paper, reviewed and approved the final manuscript.

\section{CONFLICTS OF INTEREST}

The authors declare no conflicts of interest.

\section{FUNDING}

This manuscript has been developed under the scope of the project NORTE-01-0145-FEDER-000013, supported by the Northern Portugal Regional Operational Programme (NORTE 2020) under the Portugal Partnership Agreement, through the European Regional Development Fund (FEDER), and through the Competitiveness Factors Operational Programme (COMPETE) and by National funds, through the Foundation for Science and Technology (FCT). SG received a fellowship from FCT (ref. SFRH/BPD/117858/2016).

\section{REFERENCES}

1. Balkwill F, Mantovani A. Inflammation and cancer: back to Virchow? Lancet. 2001;357(9255):539-45.

2. Pavlova NN, Thompson CB. The Emerging Hallmarks of Cancer Metabolism. Cell Metab. 2016;23(1):27-47.

3. Hanahan D, Weinberg RA. Hallmarks of cancer: the next generation. Cell. 2011;144(5):646-74.

4. Shalapour S, Karin M. Immunity, inflammation, and cancer: an eternal fight between good and evil. J Clin Invest. 2015;125(9):3347-55.

5. Coussens LM, Werb Z. Inflammation and cancer. Nature. 2002;420(6917):860-7.

6. Qu X, Tang Y, Hua S. Immunological Approaches Towards Cancer and Inflammation: A Cross Talk. Front Immunol. 2018;9:563.

7. Valkenburg KC, de Groot AE, Pienta KJ. Targeting the tumour stroma to improve cancer therapy. Nat Rev Clin Oncol. 2018;15(6):366-81.

8. O’Sullivan D, Sanin DE. Metabolic interventions in the immune response to cancer. Nat Rev Immunol. 2019;19(5):324-35.

9. DeBerardinis RJ, Chandel NS. Fundamentals of cancer metabolism. Sci Adv. 2016;2(5):e1600200.

10. Warburg O, Wind F, Negelein E. THE METABOLISM OF TUMORS IN THE BODY. J Gen Physiol. 1927;8(6):519-30.

11. Warburg O. On the origin of cancer cells. Science. 1956;123(3191):309-14.

12. Halestrap AP. The SLC16 gene family - structure, role and regulation in health and disease. Mol Asp Med. 2013;34(2-3):337-49.

13. Parks SK, Chiche J, Pouyssegur J. Disrupting proton dynamics and energy metabolism for cancer therapy. Nat Rev Cancer. 2013;13(9):611-23. 
14. Erra Diaz F, Dantas E, Geffner J. Unravelling the Interplay between Extracellular Acidosis and Immune Cells. Mediators Inflamm. 2018;2018:1218297.

15. Granja S, Tavares-Valente D, Queiros O, Baltazar F. Value of pH regulators in the diagnosis, prognosis and treatment of cancer. Semin Cancer Biol. 2017;43:17-34.

16. Pucino V, Bombardieri M, Pitzalis C, Mauro C. Lactate at the crossroads of metabolism, inflammation, and autoimmunity. Eur J Immunol. 2017;47(1):14-21.

17. Pearce EL, Pearce EJ. Metabolic pathways in immune cell activation and quiescence. Immunity. 2013;38(4):633-43.

18. Biswas SK. Metabolic Reprogramming of Immune Cells in Cancer Progression. Immunity. 2015;43(3):435-49.

19. Marchiq I, Pouyssegur J. Hypoxia, cancer metabolism and the therapeutic benefit of targeting lactate/H(+) symporters. J Mol Med. 2016;94(2):155-71.

20. Sola-Penna M. Metabolic regulation by lactate. IUBMB Life. 2008;60(9):605-8.

21. Faubert B, Li KY, Cai L, Hensley CT, Kim J, Zacharias LG, et al. Lactate Metabolism in Human Lung Tumors. Cell. 2017;171(2):358-71.e9.

22. Haas R, Smith J, Rocher-Ros V, Nadkarni S, Montero-Melendez T, D'Acquisto F, et al. Lactate Regulates Metabolic and Pro-inflammatory Circuits in Control of T Cell Migration and Effector Functions. PLoS Biol. 2015;13(7):e1002202.

23. Dienel GA. Brain lactate metabolism: the discoveries and the controversies. J Cereb Blood Flow metab. 2012;32(7):1107-38.

24. Walenta S, Mueller-Klieser WF. Lactate: mirror and motor of tumor malignancy. Semin Radiat Oncol. 2004;14(3):267-74.

25. San-Millan I, Brooks GA. Reexamining cancer metabolism: lactate production for carcinogenesis could be the purpose and explanation of the Warburg Effect. Carcinogenesis. 2017;38(2):119-33.

26. Augoff K, Hryniewicz-Jankowska A, Tabola R. Lactate dehydrogenase 5: an old friend and a new hope in the war on cancer. Cancer Lett. 2015;358(1):1-7.

27. Pinheiro C, Longatto-Filho A, Azevedo-Silva J, Casal M, Schmitt FC, Baltazar F. Role of monocarboxylate transporters in human cancers: state of the art. J Bioenerg Biomembr. 2012;44(1):127-39.

28. Ranganathan P, Shanmugam A, Swafford D, Suryawanshi A, Bhattacharjee P, Hussein MS. GPR81, a Cell-Surface Receptor for Lactate, Regulates Intestinal Homeostasis and Protects Mice from Experimental Colitis. J Immunol. 2018;200(5):1781-9.

29. Chen P, Zuo H, Xiong H, Kolar MJ, Chu Q, Saghatelian A, et al. Gpr132 sensing of lactate mediates tumor-macrophage interplay to promote breast cancer metastasis. Proc Natl Acad Sci U S A. 2017;114(3):580-5.

30. Morrot A, da Fonseca LM, Salustiano EJ, Gentile LB, Conde L, Filardy AA, et al. Metabolic Symbiosis and Immunomodulation: How Tumor Cell-Derived Lactate May Disturb Innate and Adaptive Immune Responses. Front Oncol. 2018;8:81.

31. Angelin A, Gil-de-Gomez L, Dahiya S, Jiao J, Guo L, Levine MH, et al. Foxp3 Reprograms T Cell Metabolism to Function in Low-Glucose, High-Lactate Environments. Cell Metab. 2017;25(6):1282-93.e7. 
32. Lin S, Sun L, Lyu X, Ai X, Du D, Su N, et al. Lactate-activated macrophages induced aerobic glycolysis and epithelial-mesenchymal transition in breast cancer by regulation of CCL5-CCR5 axis: a positive metabolic feedback loop. Oncotarget. 2017;8(66):110426-43.

33. Errea A, Cayet D, Marchetti P, Tang C, Kluza J, Offermanns S, et al. Lactate Inhibits the Pro-Inflammatory Response and Metabolic Reprogramming in Murine Macrophages in a GPR81-Independent Manner. PLoS One. 2016;11(11):e0163694.

34. Husain Z, Seth P, Sukhatme VP. Tumor-derived lactate and myeloid-derived suppressor cells: Linking metabolism to cancer immunology. Oncoimmunology. 2013;2(11):e26383.

35. Whitaker-Menezes D, Martinez-Outschoorn UE, Lin Z, Ertel A, Flomenberg N, Witkiewicz AK, et al. Evidence for a stromal-epithelial "lactate shuttle" in human tumors: MCT4 is a marker of oxidative stress in cancer-associated fibroblasts. Cell Cycle. 2011;10(11):1772-83.

36. Joyce JA, Fearon DT. T cell exclusion, immune privilege, and the tumor microenvironment. Science. 2015;348(6230):74-80.

37. Chang CH, Qiu J, O’Sullivan D, Buck MD, Noguchi T, Curtis JD, et al. Metabolic Competition in the Tumor Microenvironment Is a Driver of Cancer Progression. Cell. 2015;162(6):1229-41.

38. Wang T, Liu G, Wang R. The Intercellular Metabolic Interplay between Tumor and Immune Cells. Front Immunol. 2014;5:358.

39. Romero-Garcia S, Moreno-Altamirano MM, Prado-Garcia H, Sanchez-Garcia FJ. Lactate Contribution to the Tumor Microenvironment: Mechanisms, Effects on Immune Cells and Therapeutic Relevance. Front Immunol. 2016;7:52.

40. Hargadon KM. Strategies to Improve the Efficacy of Dendritic Cell-Based Immunotherapy for Melanoma. Front Immunol. 2017;8:1594.

41. Mishra D, Banerjee D. Lactate Dehydrogenases as Metabolic Links between Tumor and Stroma in the Tumor Microenvironment. Cancers. 2019;11(6). doi: 10.3390/cancers11060750

42. Sonveaux P, Vegran F, Schroeder T, Wergin MC, Verrax J, Rabbani ZN, et al. Targeting lactate-fueled respiration selectively kills hypoxic tumor cells in mice. J Clin Invest. 2008;118(12):3930-42.

43. Hoque R, Farooq A, Ghani A, Gorelick F, Mehal WZ. Lactate reduces liver and pancreatic injury in Toll-like receptor- and inflammasome-mediated inflammation via GPR81-mediated suppression of innate immunity. Gastroenterology. 2014;146(7):1763-74.

44. Iraporda C, Romanin DE, Bengoa AA, Errea AJ, Cayet D, Foligne B, et al. Local Treatment with Lactate Prevents Intestinal Inflammation in the TNBSInduced Colitis Model. Front Immunol. 2016;7:651.

45. Iraporda C, Errea A, Romanin DE, Cayet D, Pereyra E, Pignataro O, et al. Lactate and short chain fatty acids produced by microbial fermentation downregulate proinflammatory responses in intestinal epithelial cells and myeloid cells. Immunobiology. 2015;220(10):1161-9. 
46. Singer K, Cheng WC, Kreutz M, Ho PC, Siska PJ. Immunometabolism in cancer at a glance. Dis Model Mech. 2018;11(8). doi: 10.1242/dmm.034272

47. Brand A, Singer K, Koehl GE, Kolitzus M, Schoenhammer G, Thiel A, et al. LDHA-Associated Lactic Acid Production Blunts Tumor Immunosurveillance by T and NK Cells. Cell Metab. 2016;24(5):657-71.

48. Singhal S, Bhojnagarwala PS, O'Brien S, Moon EK, Garfall AL, Rao AS, et al. Origin and Role of a Subset of Tumor-Associated Neutrophils with AntigenPresenting Cell Features in Early-Stage Human Lung Cancer. Cancer Cell. 2016;30(1):120-35.

49. Shen M, Hu P, Donskov F, Wang G, Liu Q, Du J. Tumor-associated neutrophils as a new prognostic factor in cancer: a systematic review and meta-analysis. PLoS One. 2014;9(6):e98259.

50. Rao HL, Chen JW, Li M, Xiao YB, Fu J, Zeng YX, et al. Increased intratumoral neutrophil in colorectal carcinomas correlates closely with malignant phenotype and predicts patients' adverse prognosis. PLoS One. 2012;7(1):e30806.

51. Shaul ME, Fridlender ZG. Tumour-associated neutrophils in patients with cancer. Nat Rev Clin Oncol. 2019;16(10):601-20. doi: 10.1038/s41571-019-0222-4

52. Khatib-Massalha E, Kumari A, Golan K, Massalha H, Avemaria F, Gur-Cohen $\mathrm{S}$, et al. Lactate Release By Bone Marrow Neutrophils Promotes Their Inflammatory Mobilization Via Endothelial GPR81 Signaling. Blood. 2017;130(Suppl 1):446.

53. van Ravenswaay Claasen HH, Kluin PM, Fleuren GJ. Tumor infiltrating cells in human cancer. On the possible role of $\mathrm{CD} 16^{+}$macrophages in antitumor cytotoxicity. Lab Invest. 1992;67(2):166-74.

54. Van Overmeire E, Laoui D, Keirsse J, Van Ginderachter JA, Sarukhan A. Mechanisms driving macrophage diversity and specialization in distinct tumor microenvironments and parallelisms with other tissues. Front Immunol. 2014;5:127.

55. Mantovani A, Allavena P, Sica A, Balkwill F. Cancer-related inflammation. Nature. 2008;454(7203):436-44.

56. Biswas SK, Mantovani A. Orchestration of metabolism by macrophages. Cell Metab. 2012;15(4):432-7.

57. Noy R, Pollard JW. Tumor-associated macrophages: from mechanisms to therapy. Immunity. 2014;41(1):49-61.

58. Mantovani A, Sica A. Macrophages, innate immunity and cancer: balance, tolerance, and diversity. Curr Opin Immunol. 2010;22(2):231-7.

59. Richards DM, Hettinger J, Feuerer M. Monocytes and macrophages in cancer: development and functions. Cancer Microenviron. 2013;6(2):179-91.

60. Goetze K, Walenta S, Ksiazkiewicz M, Kunz-Schughart LA, Mueller-Klieser W. Lactate enhances motility of tumor cells and inhibits monocyte migration and cytokine release. Int J Oncol. 2011;39(2):453-63.

61. Dietl K, Renner K, Dettmer K, Timischl B, Eberhart K, Dorn C, et al. Lactic acid and acidification inhibit TNF secretion and glycolysis of human monocytes. J Immunol. 2010;184(3):1200-9. 
62. Langowski JL, Zhang X, Wu L, Mattson JD, Chen T, Smith K, et al. IL-23 promotes tumour incidence and growth. Nature. 2006;442(7101):461-5.

63. Shime H, Yabu M, Akazawa T, Kodama K, Matsumoto M, Seya T, et al. Tumorsecreted lactic acid promotes IL-23/IL-17 proinflammatory pathway. J Immunol. 2008;180(11):7175-83.

64. Tan Z, Xie N, Banerjee S, Cui H, Fu M, Thannickal VJ, et al. The monocarboxylate transporter 4 is required for glycolytic reprogramming and inflammatory response in macrophages. J Biol Chem. 2015;290(1):46-55.

65. Ippolito L, Morandi A, Giannoni E, Chiarugi P. Lactate: A Metabolic Driver in the Tumour Landscape. Trends Biochem Sci. 2019;44(2):153-66.

66. Colegio OR, Chu NQ, Szabo AL, Chu T, Rhebergen AM, Jairam V, et al. Functional polarization of tumour-associated macrophages by tumourderived lactic acid. Nature. 2014;513(7519):559-63.

67. Ohashi T, Akazawa T, Aoki M, Kuze B, Mizuta K, Ito Y, et al. Dichloroacetate improves immune dysfunction caused by tumor-secreted lactic acid and increases antitumor immunoreactivity. Int J Cancer. 2013;133(5):1107-18.

68. Ohashi T, Aoki M, Tomita H. M2-like macrophage polarization in high lactic acid-producing head and neck cancer. Cancer Sci. 2017;108(6):1128-34.

69. Ye H, Zhou Q, Zheng S, Li G, Lin Q, Wei L, et al. Tumor-associated macrophages promote progression and the Warburg effect via CCL18/NF-kB/VCAM-1 pathway in pancreatic ductal adenocarcinoma. Cell Death Dis. 2018;9(5):453.

70. Zhao Y, Wang D, Xu T, Liu P, Cao Y, Wang Y, et al. Bladder cancer cells reeducate TAMs through lactate shuttling in the microfluidic cancer microenvironment. Oncotarget. 2015;6(36):39196-210.

71. Banchereau J, Steinman RM. Dendritic cells and the control of immunity. Nature. 1998;392(6673):245-52.

72. Veglia F, Gabrilovich DI. Dendritic cells in cancer: the role revisited. Curr Opin Immunol. 2017;45:43-51.

73. Ma Y, Shurin GV, Peiyuan Z, Shurin MR. Dendritic cells in the cancer microenvironment. J Cancer. 2013;4(1):36-44.

74. Vermeulen M, Giordano M, Trevani AS, Sedlik C, Gamberale R, FernandezCalotti P, et al. Acidosis improves uptake of antigens and MHC class Irestricted presentation by dendritic cells. J Immunol. 2004;172(5):3196-204.

75. Chirasani SR, Leukel P, Gottfried E, Hochrein J, Stadler K, Neumann B, et al. Diclofenac inhibits lactate formation and efficiently counteracts local immune suppression in a murine glioma model. Int J Cancer. 2013;132(4):843-53.

76. Nasi A, Fekete T, Krishnamurthy A, Snowden S, Rajnavolgyi E, Catrina AI, et al. Dendritic cell reprogramming by endogenously produced lactic acid. J Immunol. 2013;191(6):3090-9.

77. Gottfried E, Kunz-Schughart LA, Ebner S, Mueller-Klieser W, Hoves S, Andreesen R, et al. Tumor-derived lactic acid modulates dendritic cell activation and antigen expression. Blood. 2006;107(5):2013-21.

78. Vivier E, Tomasello E, Baratin M, Walzer T, Ugolini S. Functions of natural killer cells. Nat Immunol. 2008;9(5):503-10. 
79. Husain Z, Huang Y, Seth P, Sukhatme VP. Tumor-derived lactate modifies antitumor immune response: effect on myeloid-derived suppressor cells and NK cells. J Immunol. 2013;191(3):1486-95.

80. Zhang C, Wang S, Yang C, Rong R. The Crosstalk between Myeloid Derived Suppressor Cells and Immune Cells: To Establish Immune Tolerance in Transplantation. J Immunol Res. 2016;2016:4986797.

81. Bruno A, Mortara L, Baci D, Noonan DM, Albini A. Myeloid Derived Suppressor Cells Interactions With Natural Killer Cells and Pro-angiogenic Activities: Roles in Tumor Progression. Front Immunol. 2019;10:771.

82. Marshall JS, Warrington R, Watson W, Kim HL. An introduction to immunology and immunopathology. Allergy Asthma Clin Immunol. 2011;7(Suppl 1):S1. doi: 10.1186/1710-1492-7-S1-S1

83. Zhu J, Paul WE. CD4 T cells: fates, functions, and faults. Blood. 2008;112(5):1557-69.

84. Asadzadeh Z, Mohammadi H, Safarzadeh E, Hemmatzadeh M, MahdianShakib A, Jadidi-Niaragh F, et al. The paradox of Th17 cell functions in tumor immunity. Cell Immunol. 2017;322:15-25.

85. Weaver CT, Harrington LE, Mangan PR, Gavrieli M, Murphy KM. Th17: an effector $\mathrm{CD} 4 \mathrm{~T}$ cell lineage with regulatory $\mathrm{T}$ cell ties. Immunity. 2006;24(6):677-88.

86. Yuseff MI, Pierobon P, Reversat A, Lennon-Dumenil AM. How B cells capture, process and present antigens: a crucial role for cell polarity. Nat Rev Immunol. 2013;13(7):475-86.

87. Palmer CS, Ostrowski M, Balderson B, Christian N, Crowe SM. Glucose metabolism regulates $\mathrm{T}$ cell activation, differentiation, and functions. Front Immunol. 2015;6:1.

88. Hirschhaeuser F, Sattler UG, Mueller-Klieser W. Lactate: a metabolic key player in cancer. Cancer Res. 2011;71(22):6921-5.

89. Lacroix R, Rozeman EA, Kreutz M, Renner K, Blank CU. Targeting tumorassociated acidity in cancer immunotherapy. Cancer Immunol Immunother. 2018;67(9):1331-48. doi: 10.1007/s00262-018-2195-Z

90. Calcinotto A, Filipazzi P, Grioni M, Iero M, De Milito A, Ricupito A, et al. Modulation of microenvironment acidity reverses anergy in human and murine tumor-infiltrating T lymphocytes. Cancer Res. 2012;72(11):2746-56.

91. Fischer K, Hoffmann P, Voelkl S, Meidenbauer N, Ammer J, Edinger M, et al. Inhibitory effect of tumor cell-derived lactic acid on human T Cells Blood. 2007;109(9):3812-9.

92. Huber V, Camisaschi C, Berzi A, Ferro S, Lugini L, Triulzi T, et al. Cancer acidity: An ultimate frontier of tumor immune escape and a novel target of immunomodulation. Semin Cancer Biol. 2017;43:74-89.

93. Broer S. Lactate transportation is required for lymphocyte activation. Nat Chem Biol. 2005;1(7):356-7.

94. Xia H, Wang W. Suppression of FIP200 and autophagy by tumor-derived lactate promotes naive $\mathrm{T}$ cell apoptosis and affects tumor immunity. Sci Immunol. 2017;2(17). doi: 10.1126/sciimmunol.aan4631 
95. Gupta S, Roy A, Dwarakanath BS. Metabolic Cooperation and Competition in the Tumor Microenvironment: Implications for Therapy. Front Oncol. 2017;7:68.

96. Siska PJ, Rathmell JC. T cell metabolic fitness in antitumor immunity. Trends Immunology. 2015;36(4):257-64.

97. Granja S, Pinheiro C, Reis RM, Martinho O, Baltazar F. Glucose addiction in cancer therapy: advances and drawbacks. Curr Drug Metab. 2015;16(3):221-42.

98. Pisarsky L, Bill R, Fagiani E, Dimeloe S, Goosen RW, Hagmann J, et al. Targeting Metabolic Symbiosis to Overcome Resistance to Anti-angiogenic Therapy. Cell Rep. 2016;15(6):1161-74.

99. Pilon-Thomas S, Kodumudi KN, El-Kenawi AE, Russell S, Weber AM, Luddy K, et al. Neutralization of Tumor Acidity Improves Antitumor Responses to Immunotherapy. Cancer Res. 2016;76(6):1381-90.

100. Guan X, Hasan MN, Begum G, Kohanbash G, Carney KE, Pigott VM, et al. Blockade of $\mathrm{Na} / \mathrm{H}$ exchanger stimulates glioma tumor immunogenicity and enhances combinatorial TMZ and anti-PD-1 therapy. Cell Death Dis. 2018;9(10):1010.

How to cite this article:

Santos N, Pereira-Nunes A, Baltazar F, Granja S. Lactate as a Regulator of Cancer Inflammation and Immunity. Immunometabolism. 2019;1:e190015. https://doi.org/10.20900/immunometab20190015 